\title{
Prevalência e fatores associados à disfunção temporomandibular em estudantes de
}

\section{fisioterapia: estudo transversal}

\author{
Prevalence and factors associated with temporomandibular disorder in physiotherapy students: \\ cross-sectional study
}

Prevalencia y factores asociados al trastorno temporomandibular en estudiantes de fisioterapia: un estudio transversal

Recebido: 12/04/2021 | Revisado: 20/04/2021 | Aceito: 26/04/2021 | Publicado: 10/05/2021

Kacielle Saraiva dos Reis
ORCID: https://orcid.org/0000-0003-0990-7752
Centro Universitário UNIFACID, Brasil
E-mail: kacielesaraiva@ outlook.com
Valéria Alves da Rocha
ORCID: https://orcid.org/0000-0003-4667-5153
Centro Universitário UNIFACID, Brasil
E-mail: valeriarocha_10@ hotmail.com
Neusa Barros Dantas Neta
ORCID: https://orcid.org/0000-0001-7127-1463
Centro Universitário UNIFACID, Brasil
E-mail: nbdn2@ msn.com
Klégea Maria Câncio Ramos Cantinho
ORCID: https://orcid.org/0000-0002-1685-5658
Centro Universitário UNIFACID, Brasil
E-mail: professoraklegea@ gmail.com
Guilherme Pertinni de Morais Gouveia
ORCID: https://orcid.org/0000-0001-6470-2341
Universidade Federal do Delta do Parnaíba, Brasil
E-mail: gpfatufpi@gmail.com
Gabriela Dantas Carvalho
ORCID: https://orcid.org/0000-0002-9571-3323
Centro Universitário UNIFACID, Brasil
E-mail: ftgabrieladantas@ @otmail.com

\section{Resumo}

O objetivo deste estudo é avaliar a prevalência e fatores associados à DTM em estudantes concludentes do curso de Fisioterapia. Trata-se de um estudo transversal, realizado com estudantes do oitavo ao décimo período, por meio da aplicação de questionários, visando identificar o perfil sociodemográfico e acadêmico, hábitos parafuncionais, severidade da DTM e o nível de ansiedade. Os dados foram analisados no programa Statistical Package for the Social Sciences Aplicou-se o Teste de Tendência Linear e considerou-se significativo valor de $\mathrm{p}<0,05$. Os dados foram expressos em tabelas. Foram avaliados 30 acadêmicos. A prevalência de DTM foi 100,0\%, com maior incidência de severidade $(66,7 \%, n=20, p=0,001)$ e ansiedade $(46,6 \%, n=14)$ moderada, com maior acometimento para o gênero feminino $(76,6 \%, n=23, p=0,039)$, manifestando sintomas de cefaleia $(70 \%, n=21)$, crepitação $(65,7$, $n=17)$ e tensão cervical $(93,3, n=28)$. A prevalência de DTM e ansiedade é alta nos estudantes concludentes de fisioterapia, manifestando-se com severidade moderada e tendo maior acometimento em estudantes do gênero feminino.

Palavras-chave: Estudantes; Fisioterapia; Disfunção temporomandibular.

\begin{abstract}
The aim of this study is to evaluate the prevalence and factors associated with TMD in students who completed the Physiotherapy course. This is a cross-sectional study, carried out with students from the eighth to the tenth period, through the application of questionnaires, aiming to identify the sociodemographic and academic profile, parafunctional habits, severity of TMD and the level of anxiety. The data were analyzed using the Statistical Package for the Social Sciences program The Linear Trend Test was applied and a significant value of $\mathrm{p}<0.05$ was considered. The data were expressed in tables. Thirty students were evaluated. The prevalence of TMD was $100.0 \%$, with a higher incidence of severity $(66.7 \%, \mathrm{n}=20, \mathrm{p}=0.001)$ and moderate anxiety $(46.6 \%, \mathrm{n}=14)$, with greater involvement in females $(76.6 \%, \mathrm{n}=23, \mathrm{p}=0.039)$, showing symptoms of headache $(70 \%, \mathrm{n}=21)$, crackling $(65.7, \mathrm{n}=17)$ and cervical tension $(93.3, \mathrm{n}=28)$. The prevalence of TMD and anxiety is high in students completing physiotherapy, manifesting itself with moderate severity and being more affected by female students.
\end{abstract}


Keywords: Students; Physiotherapy; Temporomandibular dysfunction.

\section{Resumen}

El objetivo de este estudio es evaluar la prevalencia y los factores asociados a TTM en estudiantes que completaron el curso de Fisioterapia. Se trata de un estudio transversal, realizado con estudiantes del octavo al décimo período, mediante la aplicación de cuestionarios, con el objetivo de identificar el perfil sociodemográfico y académico, los hábitos parafuncionales, la gravedad de los TTM y el nivel de ansiedad. Los datos se analizaron mediante el paquete estadístico para el programa de Ciencias Sociales, se aplicó el Test de Tendencia Lineal y se consideró un valor significativo de $\mathrm{p}<0.05$. Los datos se expresaron en tablas. Se evaluaron treinta estudiantes. La prevalencia de TTM fue del $100,0 \%$, con mayor incidencia de severidad $(66,7 \%, \mathrm{n}=20, \mathrm{p}=0,001)$ y ansiedad moderada $(46,6 \%, \mathrm{n}=14)$, con mayor afectación en mujeres $(76,6 \%, n=23, p=0,039)$, mostrando síntomas de cefalea $(70 \%, n=21)$, crepitaciones $(65,7, \mathrm{n}=17)$ y tensión cervical $(93,3, \mathrm{n}=28)$. La prevalencia de TTM y ansiedad es alta en los estudiantes que completan fisioterapia, manifestándose con gravedad moderada y siendo más afectados por las estudiantes mujeres.

Palabras clave: Estudiantes; Fisioterapia; Disfunción temporomandibular.

\section{Introdução}

A articulação temporomandibular (ATM) é um dos componentes do sistema estomatognático, constituída por diversas estruturas internas e externas. Ela é capaz de produzir importantes movimentos, que envolvem funções como a mastigação, fonação, deglutição e postura (Gonzalez, 2008).

Diante das desordens que prejudicam a ATM, diversas complicações clínicas são percebidas e comprometem os músculos da mastigação e as estruturas relacionadas, como a própria ATM (Alves, 2010). Com isso, pode ocorrer o aparecimento de algumas sintomatologias cujas características são: dores crônicas, fadiga, sensibilidade, restrição do movimento e estalidos/crepitação, promovendo um desequilíbrio biomecânico nesta região, com o desenvolvimento de dor orofacial, desorganização muscular, neuralgias, travamentos e cefaleias, seguidas de dores na região cervical (Gonzalez, 2008).

Estes sinais e sintomas, não são, obrigatoriamente, uma condição da doença ou que necessitará de uma abordagem terapêutica e, posteriormente, um tratamento. Todavia, Torres et al. (2012) consideram que de 5\% a 20\% dos sujeitos que apresentam a sintomatologia deverão desenvolver lesões na ATM, conduzindo a um quadro de disfunção temporomandibular (DTM) (Basso et al., 2010).

A etiologia da DTM é multifatorial, estando relacionada com diversos fatores, dentre eles: má oclusão, traumas na região da mandíbula, alterações dos músculos mastigatórios, desequilíbrio postural, além de problemas emocionais (Chaves et al., 2007). Quando associado ao fator anatômico é comum observar a presença dos hábitos parafuncionais como a onicofagia, bem como aos distúrbios da marcha, sobrecarga osteomioarticular, poucas horas de sono e a má ergonomia dos assentos e calçados (Bezerra et al., 2012).

O estudo epidemiológico de Bender (2014) mostra a presença de sinais e sintomas de DTM em todas as faixas etárias, com prevalência estimada entre 3 e 15\% da população. Dentre os grupos mais afetados, destacam-se aqueles que, associado às disfunções anatômicas e biomecânicas, apresentam alto grau de ansiedade e sobrecarga de tensão, tais como os estudantes de ensino superior, em especial, aqueles que se encontram no final da graduação Nesta fase os estudantes estão, em sua maioria, em uma rotina extensa de aulas, estágios curriculares, produção do trabalho de conclusão de curso (TCC), bem como a preocupação com o ingresso no mercado de trabalho (Cauás, 2004).

A literatura mostra escassez de estudos que associem as atividades acadêmicas com o desenvolvimento da DTM (Bezerra et al., 2012). Tendo em vista a importância do diagnóstico precoce, buscou-se identificar a prevalência e os sintomas que podem estar envolvidos em indivíduos jovens e não pacientes. Tais dados podem favorecer o diagnóstico precoce, bem como direcionar o tratamento em intervenções futuras.

Diante disso, o objetivo desse estudo é avaliar a prevalência e a severidade da DTM, nível de ansiedade e os fatores associados à DTM em estudantes concludentes do curso de Fisioterapia de um Centro Universitário de Teresina-PI. 


\section{Metodologia}

\subsection{Princípios éticos}

A presente pesquisa foi realizada de acordo com as diretrizes para pesquisa envolvendo humanos preconizadas pela Resolução do Conselho Nacional de Saúde 466/12, sendo a mesma submetida à apreciação do Comitê de Ética em Pesquisa com Seres Humanos do Centro Universitário UNIFACID e aprovada sem restrições sob o número de parecer 2.848.331. Todos os voluntários validaram a sua participação na pesquisa por meio da assinatura no termo de consentimento livre esclarecido (TCLE).

\section{2,2 Desenho e participantes}

Trata-se de um estudo observacional, analítico de corte transversal, realizado em estudantes do oitavo ao décimo período do curso de Fisioterapia de um Centro Universitário de Teresina-PI, no período de agosto a dezembro de 2019. O estudo foi conduzido no serviço escola de Fisioterapia, local que dispõe do espaço físico necessário ao desenvolvimento do trabalho.

A amostra foi selecionada por conveniência a partir da lista obtida junto à coordenação do respectivo curso. No primeiro e segundo semestre de 2019, havia 52 estudantes matriculados entre o oitavo e décimo período do curso de Fisioterapia do referido Centro Universitário. Após convite para a participação na pesquisa e aplicação dos critérios de exclusão, a pesquisa contou com uma amostra de 30 participantes.

Foram incluídos estudantes independentes do gênero, sem restrições de faixa etária, que estivessem cumprindo todas as atividades acadêmicas (aulas, estágios e realização do TCC). Foram excluídos os alunos que usavam prótese dentária, que apresentavam comprometimento neuromotor, fraturas, cirurgia prévia ou má formação orofacial, com diagnóstico de paralisia facial ou trauma facial e/ou que estariam sob tratamento fisioterápico, fonoaudiológico e/ou odontológico.

\subsection{Instrumentos de Avaliação}

Os questionários foram lidos, interpretados e respondidos pelos voluntários, a fim de evitar qualquer influência sobre as respostas. A pesquisa foi realizada na forma de entrevista com aplicação de questionário dividido em duas seções. A primeira seção continha perguntas como: idade, gênero, período matriculado, quantidade de disciplinas realizadas, tempo de atividades acadêmicas, presença de hábitos parafuncionais, sintomas, bem como o estado emocional. A segunda seção foi composta por questionários validados e não invasivos que avaliam diagnóstico e a severidade dos voluntários com DTM e os diferentes níveis de ansiedade dos acadêmicos.

Para o diagnóstico e a severidade da DTM foi aplicado o Questionário Anamnésico de Fonseca, composto por 10 questões, com três tipos de respostas e pontuações (sim/10, às vezes/5 e não/0). Ao somar os pontos, pode-se classificar os voluntários em diferenciadas classificações quanto ao agravamento e aos sintomas, tais como: ausência da DTM de 0 a 15 pontos, de grau leve de 20 a 40 pontos, moderado 45 a 65 pontos e por último de grau severo de 70 a 100 pontos (Chaves et al., 2008).

Para avaliar os diferentes níveis de ansiedade dos acadêmicos foi aplicado o questionário do Inventário de Ansiedade Traço-Estado (IDATE), traduzida e validada por Spielberger, Gorsuch e Lushene (Biaggio \& Natalício, 1979). O mesmo é formado por duas escalas de auto relatório que avaliam a ansiedade quanto ao estado (IDATE-E) e traço (IDATE-T). Cada situação possui 20 perguntas objetivas com pontuação de 1 a 4, para cada pergunta é atribuída a pontuação que corresponde a resposta, porém, para as perguntas com caráter positivo a pontuação é invertida, ou seja, se a resposta for 4, sua pontuação será 1. O escore varia de 20 a 80 pontos, no qual o total dos pontos somados pelos participantes resultou em um grau de 
classificação de ansiedade, podendo ser: ansiedade baixa (20 a 34 pontos); moderada (35 de 49 pontos); grave (50 a 64 pontos); e em pânico (65 a 80 pontos) (Fioravanti et al., 2006).

\subsection{Análise Estatística}

Os dados foram analisados no programa Statistical Package for the Social Sciences - SPSS, versão 21.0. A análise descritiva foi realizada e os dados apresentados na forma de frequência, porcentagem, média e desvio padrão. Foi realizado o Teste de Tendência Linear para associar DTM e ansiedade com fatores sociodemográficos, considerou-se valor de $\mathrm{p}<0,05$ como significante. Os dados foram expressos em forma de tabelas.

\section{Resultados}

A amostra foi composta por 30 estudantes matriculados do oitavo ao décimo período do curso de Fisioterapia. Dentre os estudantes, 76,7\% ( $n=23)$ eram do gênero feminino e 23,3 ( $n=7)$ masculino, apresentando idade entre 19 a 26 anos, com uma média de $\pm 22,5$ anos, 50,0\% ( $\mathrm{n}=15$ ) naturais do estado do Piauí, porém, de municípios distintos de Teresina, de tal forma que $80 \%(n=24)$ dos entrevistados relataram compartilhar moradia com amigos ou familiares e que consideravam apresentar boa relação interpessoal $(76,7 \%, \mathrm{n}=23)$. Ao questionar se exerciam atividade profissional associado à vida acadêmica, $90 \%$ ( $\mathrm{n}=27)$ afirmaram que não, uma vez que o curso apresenta atividades em regime integral (Tabela 1).

Quanto às atividades acadêmicas que os voluntários cursavam, com base no projeto pedagógico do curso de Fisioterapia da referida instituição, os alunos a partir do oitavo período começam a realizar o estágio obrigatório de 320 horas, associado à realização do TCC 1 e 2 no 9 e 10 períodos, respectivamente. Logo, dentro da população investigada, 100\% (n=30) encontravam-se cursando o estágio associado às disciplinas teóricas e apenas $60 \%(\mathrm{n}=18)$ estavam cursando disciplinas teóricas, estágio e TCC. Quando questionados a respeito da quantidade dos componentes curriculares realizadas, 6 (20\%) realizavam até 5 disciplinas e 24 ( $80 \%$ ) estavam cursando acima de 5 disciplinas, com uma média total de $\pm 7,4$ disciplinas para o presente semestre. $86,66 \%(\mathrm{n}=26)$ dos alunos relataram realizar atividades integrais no curso de fisioterapia, destes 8 $(26,66 \%)$ afirmaram permanecer os dois turnos na instituição, seguido dos 18 (60\%) que permaneciam nos 3 turnos (Tabela 1).

Tabela 1 - Características descritivas dos estudantes do curso de Fisioterapia, Teresina, 2019, n=30.

\begin{tabular}{lll}
\hline Variáveis & n & \% \\
\hline Gênero & 23 & 76,7 \\
Feminino & 7 & 23,3 \\
Masculino & & \\
Naturalidade & 15 & 50,0 \\
Piauí & 13 & 43,3 \\
Maranhão & 2 & 6,7 \\
Pernambuco & & \\
Ativamente laboral & 3 & 10,0 \\
Sim & 27 & 90,0 \\
Não & & \\
Atividades acadêmicas & 0 & 0 \\
Apenas disciplinas teóricas & 30 & 100 \\
Estágio obrigatório & 18 & 60 \\
Trabalho de conclusão de curso & & 20 \\
Número de disciplinas & 6 & 80 \\
Até 5 & 24 & 13,33 \\
Acima de 5 & & 86,66 \\
Turnos & 4 & 13,33 \\
Parcial (1 turno) & 26 & 26,66 \\
Integral & & 60 \\
Tempo de atividade acadêmica & 4 & \\
3 horas (1 turno) & 8 & \\
6 horas (2 turnos) & 18 & \\
Acima de 6 horas (3 turnos) & & \\
\hline
\end{tabular}

Legenda: $\mathrm{n}=$ Número de participantes; $\%=$ percentual. Fonte: Autores. 
Dentre os sinais e sintomas manifestados característicos de diagnóstico de DTM, houve predominância para cefaleia com $70 \%(n=21)$ dos entrevistados acometidos, seguido de a presença de crepitação articular ao abrir a boca $(65,7, \mathrm{n}=17) \mathrm{e}$ tensão na cervical $(93,3, \mathrm{n}=28)$ (Tabela 2).

Tabela 2 - Características sintomatológicas apresentadas pelos acadêmicos do curso de Fisioterapia, Teresina, 2019, n=30.

\begin{tabular}{lll}
\hline Dor de cabeça & n & \% \\
\hline Sim & 21 & 70,00 \\
Não & 9 & 30,00 \\
Estalidos/crepitação & & \\
Sim & 17 & 56,7 \\
Não & 13 & 43,3 \\
Limitação ao abrir a boca & & \\
Sim & 4 & 13,3 \\
Não & 26 & 86,7 \\
Tensão muscular cervical & & \\
Sim & 28 & 93,3 \\
Não & 2 & 6,7 \\
Sensibilidade & & 23,3 \\
Sim & 7 & 76,6 \\
Não & 23 & 100,0 \\
\hline Total & 30 &
\end{tabular}

Legenda: $\mathrm{n}=$ Número de participantes; $\%=$ percentual. Fonte: Autores.

Ao investigar a prática dos hábitos parafuncionais, observa-se a presença de todas as variáveis, com destaque para o dormir sobre a face $(93,3 \%, \mathrm{n}=28)$, apoiar o queixo sobre a mão $(76,6 \%)$, mascar chiclete $(73,3 \%, \mathrm{n}=23)$ e roer as unhas $(53,3 \%, \mathrm{n}=16)$ (Tabela 3$)$.

Tabela 3 - Hábitos parafuncionais realizados pelos acadêmicos do curso de Fisioterapia, Teresina, 2019, n=30.

\begin{tabular}{lcc}
\hline \multicolumn{1}{c}{ Variáveis } & n & \% \\
\hline Masca chiclete & 22 & 73,3 \\
Sim & 8 & 26,7 \\
Não & & \\
Morde objetos rígidos & 11 & 36,7 \\
Sim & 19 & 63,3 \\
Não & & \\
Range os dentes & 4 & 13,3 \\
Sim & 26 & 86,7 \\
Não & & \\
Dorme sobre a face & 28 & 93,3 \\
Sim & 2 & 6,7 \\
Não & & \\
Roer as unhas & 16 & 53,3 \\
Sim & 14 & 46,7 \\
Não & & \\
Apoia a mão no queixo & 23 & 76,7 \\
Sim & 7 & 23,3 \\
Não & 30 & 100,0 \\
\hline Total & & \\
\hline
\end{tabular}

Legenda: $\mathrm{n}=$ Número de participantes; \%= percentual. Fonte: Autores.

$\mathrm{Na}$ Tabela 4 pode-se analisar que a severidade da DTM nos acadêmicos, classificando-a em leve, moderada e severa. A DTM apresenta-se presente em 100\% ( $\mathrm{n}=30)$ dos acadêmicos investigados, sendo 16,7\% ( $\mathrm{n}=5)$ DTM leve, 66,7\% $(\mathrm{n}=20)$ DTM moderada e $16,7 \%(n=5)$ DTM grave. Nestes estudantes, apenas o gênero foi associado à DTM (valor de p=0,001), de forma que, os homens tiveram maior prevalência de DTM leve $(57,1 \%, \mathrm{n}=4)$ e as mulheres de DTM moderada $(82,6 \%, \mathrm{n}=19)$. 
Tabela 4 - Associação entre o grau de severidade da DTM fatores sociodemográficos de acadêmicos do curso de Fisioterapia, Teresina, 2019, $\mathrm{n}=30$.

\begin{tabular}{|c|c|c|c|c|c|}
\hline \multirow[b]{2}{*}{ Variáveis } & & \multicolumn{3}{|c|}{ Grau de DTM } & \multirow[t]{2}{*}{ p valor } \\
\hline & & DTM leve & DTM moderada & DTM severa & \\
\hline \multirow{4}{*}{ Gênero } & \multirow{2}{*}{ Feminino } & 1 & 19 & 3 & \\
\hline & & $4,3 \%$ & $82,6 \%$ & $13,0 \%$ & 0,001 \\
\hline & \multirow{2}{*}{ Masculino } & 4 & 1 & 2 & \\
\hline & & $57,1 \%$ & $14,3 \%$ & $28,6 \%$ & \\
\hline \multirow{2}{*}{ Total } & & 5 & 20 & 5 & \\
\hline & & $16,7 \%$ & $66,7 \%$ & $16,7 \%$ & \\
\hline
\end{tabular}

Legenda: DTM= Disfunção Temporomandibular; *p valor estatisticamente significante (Teste de Tendência Linear). Fonte: Autores.

A análise do nível de ansiedade é apresentada nas Tabelas 5 e 6, respectivamente. A Tabela 5 mostra predomínio de ansiedade baixa a grave entre os estudantes, com um índice de 26,7\% ( $n=8)$ apresentando ansiedade leve, seguido de 46,6\% $(n=14)$ com ansiedade moderada e 23,3\% ( $n=7)$ grave, tendo o gênero feminino predominante para todos os níveis de ansiedade $(76,6 \%, n=23, p=0,039)$. Enquanto o gênero masculino apresentou predominância apenas para o estado de pânico $(3,3 \%, n=1)$.

Tabela 5 - Associação entre o estado de ansiedade e fatores sociodemográficos de acadêmicos do curso de Fisioterapia, Teresina, 2019, $\mathrm{n}=30$.

\begin{tabular}{|c|c|c|c|c|c|c|}
\hline \multirow[t]{2}{*}{ Variáveis } & & \multicolumn{4}{|c|}{ IDATE I - Estado de ansiedade } & \multirow[t]{2}{*}{ p valor } \\
\hline & & Baixa & Moderada & Grave & Pânico & \\
\hline \multirow{5}{*}{ Gênero } & \multirow{2}{*}{ Feminino } & 8 & 11 & 4 & 0 & 0,039 \\
\hline & & $34,8 \%$ & $47,8 \%$ & $42,9 \%$ & $14,3 \%$ & \\
\hline & \multirow{3}{*}{ Masculino } & 0 & 3 & 3 & 1 & \\
\hline & & $0,0 \%$ & $42,9 \%$ & $17,4 \%$ & $0,0 \%$ & \\
\hline & & $16,7 \%$ & $66,7 \%$ & $0,0 \%$ & $16,7 \%$ & \\
\hline \multirow{2}{*}{ Total } & & 8 & 14 & 7 & 1 & \\
\hline & & $26,7 \%$ & $46,7 \%$ & $23,3 \%$ & $3,3 \%$ & \\
\hline
\end{tabular}

Legenda: *p valor estatisticamente significante (Teste de Tendência Linear). Fonte: Autores.

Na parte II do inventário de ansiedade IDATE-TRAÇO é apresentado na Tabela 6, no qual observa-se uma redução dos níveis de ansiedade em 20\%, comparado aos níveis de ansiedade apresentados na Tabela 5, com aumento do percentual moderado $(53,3 \%, n=16)$, mantendo o percentual de ansiedade grave $(23,3 \%, n=7)$ e pânico $(3,3 \%$, $n=1)$. Concomitante ao apresentado no inventário de ansiedade IDATE-ESTADO, houve predomínio para o gênero feminino para os níveis de ansiedade leve, moderado e grave $(76,6 \%, n=23, p=0,083)$. Não houve associação entre o traço de ansiedade e fatores sociodemográficos de acadêmicos do curso de fisioterapia. 
Tabela 6 - Associação entre o traço de ansiedade e fatores sociodemográficos de acadêmicos do curso de Fisioterapia, Teresina, 2019, $\mathrm{n}=30$.

\begin{tabular}{|c|c|c|c|c|c|c|}
\hline \multirow[t]{2}{*}{ Variáveis } & & \multicolumn{4}{|c|}{ IDATE II - Traço de ansiedade } & \multirow[t]{2}{*}{ p valor } \\
\hline & & Baixa & Moderada & Grave & Pânico & \\
\hline \multirow{4}{*}{ Gênero } & \multirow{2}{*}{ Feminino } & 6 & 13 & 4 & 0 & 0,083 \\
\hline & & $26,1 \%$ & $56,5 \%$ & $42,9 \%$ & $14,3 \%$ & \\
\hline & \multirow{2}{*}{ Masculino } & 0 & 3 & 3 & 1 & \\
\hline & & $0,0 \%$ & $42,9 \%$ & $17,4 \%$ & $0,0 \%$ & \\
\hline \multirow{2}{*}{ Total } & & 6 & 16 & 7 & 1 & \\
\hline & & $20,0 \%$ & $53,3 \%$ & $23,3 \%$ & $3,3 \%$ & \\
\hline
\end{tabular}

Legenda: *p valor estatisticamente significante (Teste de Tendência Linear). Fonte: Autores.

\section{Discussão}

Diversos estudos são conduzidos visando analisar a DTM em diversas populações com diferentes atividades e faixas etárias (Nekora-Azak et al., 2006; Bontempo \& Zavanelli, 2009), no entanto, dado ao caráter da idade e sua alta capacidade de recuperação, pouco se é discutido a respeito do desenvolvimento da DTM sobre indivíduos jovens. No entanto, deve-se considerar que é na faixa de 13 a 35 anos que o índice de DTM é o mais preponderante, isso é justificado pelas inúmeras mudanças físicas, emocionais, hormonais características desta faixa etária, bem como, das exigências profissionais impostas, como a formação acadêmica e a inserção no mercado profissional (Bove et al., 2005).

Pinto et al., (2017) descrevem que a formação acadêmica promove uma exigência maior se comparado ao ensino médio, isso deve-se ao aumento da demanda das atividades exigidas, bem como aos prazos para a realização das mesmas. A vida do estudante, em especial, daqueles que se encontram cursando os últimos períodos da graduação, é mais propensa a ser sobrecarregada em virtude das maiores responsabilidades adquiridas, deixando-os expostos as maiores reações emocionais (Sousa et al., 2016).

Diante disso, ao analisar a prevalência da DTM nos estudantes de Fisioterapia, observa-se que o gênero feminino foi o mais afetado, no entanto, deve-se considerar o perfil do grupo investigado, onde há uma maior proporcionalidade do gênero feminino na amostra, como mostra a Tabela 1, favorecendo que a predominância para o gênero dentre os afetados. Contudo, a literatura confirma que o gênero feminino tende a desencadear mais DTM (Ferreira et al., 2012; Ferreira et al., 2016). Miyazaki \& Yamamoto (2009) descrevem que a prevalência da DTM no gênero feminino pode ser explicada devido ao fato de que nessa população exista uma maior incidência de frouxidão ligamentar, o que pode acarretar maiores problemas quanto a estabilidade da ATM, bem como a influência do estado hormonal, dado pelos hormônios sexuais, especialmente o estrogênio, que desencadeia uma sensibilidade dolorosa, inclusive nos músculos mastigatórios e na patogênese da DTM, podendo o limiar de dor e a tolerância à mesma variarem de acordo com a fase do ciclo menstrual. Segundo Bereiter (2011) o estrógeno é um fator de risco para dores craniofaciais, com ação periférica e central na modulação da dor, bem como seus receptores regulam a sensibilização dos neurônios trigeminais ou exercem alguma influência nas vias trigeminais da dor.

A manifestação dos sinais e sintomas são indicativos de desgaste anatômico e/ou desalinhamento biomecânico, que se não corrigidos precocemente, podem conduzir a lesões mais graves, necessitando de correção cirúrgica. $\mathrm{O}$ guia da Academia Americana de Dor Orofacial estima que 40 a $75 \%$ da população apresenta algum sinal de DTM e que 33\% apresenta algum sintoma (Okeson, 1996). A Tabela 2 descreve os sinais e sintomas analisados com destaque para as cefaleias, estalidos/crepitação e a tensão cervical. Alves (2010) descreve que a ATM, por ser uma articulação triaxial, é capaz de realizar movimentos em todos os eixos, esta acaba sendo facilmente sobrecarregada, em especial, pelo desgaste do disco articular, 
podendo haver o contato entre as superfícies ósseas, que culminam na crepitação, seguida de dor. Essa sobrecarga sobre a ATM pode ser desencadeada por múltiplos fatores, dentre eles a tensão muscular, como relatam os estudantes, em especial, na região cervical, nos quais estão intimamente relacionados ao centro de equilíbrio muscular da cabeça e de todas as estruturas envolvidas (Basso et al., 2010). Corroborando com este estudo, Ferreira et al. (2012) ao analisarem sinais e sintomas de DTM entre homens e mulheres citam a cefaleia como a principal queixa dos avaliados, seguido das dores no pescoço e nos ombros e dores nos músculos faciais, justificando a possível correlação da cefaleia às tensões e dores musculares.

Os hábitos parafuncionais, como um dos fatores desencadeantes da DTM, são movimentos simples e repetitivos realizados de forma inconsciente e que estão associados a uma atividade inadequada ou estado emocional. Dentre os achados mais frequentes que estão associados às atividades acadêmicas destacam-se o bruxismo, onicofagia, apoio mandibular com as mãos e/ou morder o lábio (Miyazaki \& Yamamoto, 2009). A Tabela 3 citam o mascar chiclete, dormir sobre a face, apoiar a mão no queixo e roer as unhas como um dos fatores desencadeantes de DTM nos acadêmicos de Fisioterapia. Pinto et al. (2017) ao investigarem a correlação dos hábitos parafuncionais ao desenvolvimento da DTM evidenciam o apoio da mão ao queixo como um dos principais fatores desencadeantes. De forma similar, Sousa et al. (2016) realçam o hábito de dormir sobre um lado da face e o apoio da mão ao queixo, corroborando com os dados obtidos neste estudo.

Quanto aos achados relacionados ao grau de severidade da DTM, a Tabela 4 mostra maior prevalência de DTM em grau moderada. Os dados mostram divergência aos apresentados na literatura, no qual estudos voltados à análise da severidade dos acadêmicos mostram prevalência para o grau leve (Azevedo et al., 2012; Sousa et al., 2016), como mostra o estudo realizado por Bezerra et al. (2012) que ao analisarem a prevalência da DTM e de diferentes níveis de ansiedade em estudantes universitários descrevem que 48,2\% manifestam DTM em grau leve, seguido de moderado (11,3\%) e grave (3\%). Contudo, em ambos os estudos a população estudada abrange todos os períodos acadêmicos, o que permite enquadrar um percentual maior de acadêmicos com uma demanda de atividades menores, quando comparados aos concludentes que já vem em uma sequência de atividades acadêmicas ao longo dos 5 anos de curso, como os analisados no presente trabalho.

O estado emocional é um dos principais desencadeadores de tensão muscular, levando ao desenvolvimento das desordens musculoesqueléticas. Inúmeros estudos correlacionam a ansiedade e depressão com o desenvolvimento da DTM (Bezerra et al., 2012; Sousa et al., 2016). A ansiedade, apesar de ser um fenômeno universal, vivenciado por boa parcela da população mundial, ainda não possui uma definição exata, sendo a mais utilizada como uma emoção caracterizada por um estado de alerta ou tensão, fisicamente exaustivo, focalizado em um perigo ou emergência iminente e inevitável (Nunes et al., 2016).

No estudo da ansiedade são citados dois conceitos distintos: a ansiedade-estado (IDATE-E), na qual avalia um estado emocional transitório, caracterizado por sentimentos subjetivos de tensão que podem variar em intensidade ao longo do tempo, e a ansiedade-traço (IDATE-T), a qual se refere a uma disposição pessoal, relativamente estável, a responder com ansiedade a situações estressantes e uma tendência a perceber um maior número de situações como ameaçadoras (Spielberger et al., 1970; Nunes et al., 2016).

A aplicação dos inventários IDATE-E e o IDATE-T apresentaram a presença da ansiedade para todos os níveis (leve ao pânico) como mostram as Tabelas 5 e 6, respectivamente. Considerando a definição de traço de ansiedade, pode-se dizer que a maioria dos alunos tem tendência moderada de apresentar elevações dos níveis de ansiedade em situações ameaçadoras, tais como no estágio, realização de provas e apresentação do TCC. Em ambos os questionários prevaleceu maiores escores para o gênero feminino, achado este que entra em consonância com outros estudos envolvendo o IDATE (Gama et al., 2008; Santos \& Galdeano, 2009).

Acredita-se que o predomínio do estado ansioso para todos os níveis seja dado as demandas impostas aos estudantes. Observa-se que os estudantes que apresentaram ansiedade em estado grave e pânico eram os que se encontravam no nono e 
décimo período acadêmico, respectivamente, dado este que se assemelha ao encontrado no estudo de Sousa et al. (2016), no qual aplicaram o inventaram IDATE em universitários, mostrando prevalência de ansiedade em grau moderado em mais de 95\% dos pesquisados e que estes foram diagnosticados com DTM. Lima et al. (2017) analisaram o grau de ansiedade em acadêmicos concludentes do curso de enfermagem, mostrando a presença de ansiedade em níveis altos para 52,9\%, sendo estes, em sua maioria, do gênero feminino $(92,16 \%)$ e que $56,9 \%$ estavam envolvidos em atividades extracurriculares, tais como estágio, pesquisa, extensão e monitorias, o que mostra consonância com o perfil dos acadêmicos do curso de Fisioterapia investigados neste estudo.

\section{Conclusão}

Os estudantes de Fisioterapia apresentam diagnóstico sintomatológico para DTM, com maior prevalência para o gênero feminino e grau de severidade moderado. Os dados sugerem que a presença da DTM esteja correlacionada com a demanda acadêmica extensa, a realização dos hábitos parafuncionais e estado de ansiedade relatado pelos estudantes.

As observações realizadas neste estudo limitaram-se aos estudantes concludentes do curso de Fisioterapia, o que permitiu a participação de um $\mathrm{n}$ amostral pequeno. O tema abordado é amplo e acredita-se que há muito o que se explorar, visando uma análise mais apurada do impacto do ensino superior sobre a ATM. Logo, diante dos dados apresentados, no qual traz a reflexão dos impactos apresentados, bem como, a ausência de ensaios clínicos sobre a temática na literatura, visa-se novos estudos que abordem uma intervenção precoce, como forma de prevenir o desencadeamento da DTM em estudantes de ensino superior, assim como, a intervenção da fisioterapia na reabilitação dos casos identificados, visando o não agravamento dos mesmos.

\section{Referências}

Alves, R. L. B. R. (2010). A eficácia dos recursos fisioterapêuticos no ganho da amplitude de abertura bucal em pacientes com disfunções craniomandibulares. Rev Odontol UNESP, 39(1), 55-61.

Azevedo, A. B. F., Rocha, C. O. M., Resende, C. M. B. M., \& Oliveira, A. G. R. C. (2012). Prevalência de Disfunção Temporomandibular e seus diferentes níveis de severidade em estudantes de odontologia da Universidade Federal do Rio Grande do Sul. Revista Extensão \& Sociedade, 4, $235-42$. https://doi.org/10.1590/S1806-00132012000300008.

Basso, D., Corrêa, E., \& Silva, A. M. (2010). Efeito da reeducação postural global no alinhamento corporal e nas condições clínicas de indivíduos com disfunção temporomandibular associada a desvios posturais. Fisioterapia e Pesquisa, 17(1), 63-8. https://doi.org/10.1590/S1809-29502010000100012

Bender, S. D. (2014). Orofacial pain and headache: a review and look at the commonalities. Curr Pain Headache Rep., 18(400), 1-6. https://doi.org/110.1007/s11916-013-0400-5.

Bereiter, D. A., \& Okamoto, K. (2011). Neurobiology of estrogen status in deep craniofacial pain. Int Rev Neurobiol., 97, 251-84. https://doi.org/10.1016/B978-0-12-385198-7.00010-2.

Bezerra, B. P. N., Ribeiro, A. I. A. M., De Farias, A. B. L., De Farias, A. B. L., Fontes, L. B. C., \& Nascimento, S. R. (2012). Prevalência da disfunção temporomandibular e de diferentes níveis de ansiedade em estudantes universitários. Rev Dor, 13(3), 235-42. https://doi.org/10.1590/S180600132012000300008 .

Biaggio, A. M. B., \& Natalício, L. (1979). Manual para o Inventário de Ansiedade Traço-Estado (IDATE). CEPA.

Bontempo, K. V., \& Zavanelli R. A. (2009). Fatores etiológicos correlacionados à desordem temporomandibular em pacientes portadores de próteses totais bimaxilares: uma análise comparativa. Revista Gaúcha de Odontologia, 57, 67-75.

Bove, S. R. K., Guimarães, A. S., \& Smith, R. L. (2005). Caracterização dos pacientes de um ambulatório de disfunção temporomandibular e dor orofacial. Rev Lat Am Enfermagem, 13, 686-91.

Cauás, M. (2004). Incidências de hábitos parafuncionais e posturais em pacientes portadores de disfunção da articulação craniomandibular. Revista de Cirurgia e Traumatologia Buco-Maxilo-Facial, 4 (2), 121-9.

Chaves, T. C., Oliveira, A. S., \& Grossi, D. B. (2007). Principais instrumentos para avaliação da disfunção temporomandibular, parte I: índices e questionários; uma contribuição para a prática clínica e de pesquisa. Fisioterapia e pesquisa, 15(1), 92-100. http://dx.doi.org/10.1590/S180929502008000100015. 
Research, Society and Development, v. 10, n. 5, e37710514984, 2021

(CC BY 4.0) | ISSN 2525-3409 | DOI: http://dx.doi.org/10.33448/rsd-v10i5.14984

Ferreira, C. L. P., Da Silva, M. A. M. R., \& Felício, C. M. (2012). Sinais e sintomas de desordem temporomandibular em mulheres e homens. Arq Odontol., 48(1), 13-18. http://dx.doi.org/10.1590/2317-1782/20162014218

Ferreira, F. B., Da Cruz, L. M. P., Urban, V. M., Fernandes, F., Campanha, N. H., \& Jorge, J. H. (2016). Prevalência das desordens temporomandibulares em graduandos da Universidade Estadual de Ponta Grossa. Arquivos em Odontologia, 28(1), 17-21.

Fioravanti, A. C. M., Santos, L. F., Maissonette, S., Cruz, A. P. M., \& Landeira-Fernandez, J. (2006). Avaliação da estrutura fatorial da Escala de AnsiedadeTraço do IDATE. Avaliação Psicológica, 5(2), 217-24.

Gama, M. M. A., Moura, G. S., Araújo, R. F., \& Silva FT. (2008). Ansiedade-traço em estudantes universitários de Aracaju (SE). Rev Psiquiatr 30(1), 19-24.

Gonzalez, D. A. (2008). Correlação entre disfunção temporomandibular, postura e qualidade de vida. Rev. Bras. Crescimento Desenvol. Hum, 18(1), 79-86.

Lima, B. V. D. B. G., Trajano, F. M. P., Neto, G. C., Alves, R. S., Farias, J. A., \& Braga, J. E. F. (2017). Avaliação da ansiedade e autoestima em concluintes do curso de graduação em enfermagem. Rev. enferm. UFPE on-line. 11(11), 4326-33.

Miyazaki, R., \& Yamamoto, T. (2009). Sex and/or gender differences in pain. Masui, 58(1), 34-9.

Nekora-Azak, A., Evlioglu, G., Ordulu, M., \& Ișsever, H. (2006). Prevalence of symptoms associated with temporomandibular disorders in Turkish population. J Oral Rehabil, 33, 81-4. http://dx.doi.org/10.1111/j.1365-2842.2006.01543.x.

Nunes, J. C., Silva, N. C., Dantas, R. L., Vieira, T. C., \& Silva, P. A. L. (2016). Prevalência de sintomatologia para a disfunção temporomandibular (DTM) em estudantes de fisioterapia. Revista Eletrônica de Trabalhos Acadêmicos, 1(1), 1-20. http://dx.doi.org/10.1590/S1806-00132012000300008

Okeson, J. P. (1996). Orofacial pain. Guidelines for assessment, diagnosis and management. Quintessence Publishing.

Pinto, R. G. S., Leite, W. M. A., Sampaio, L. S., \& Sanchez, M. O. (2017). Associação entre sinais e sintomas de disfunção temporomandibular com depressão em universitários: estudo descritivo. Rev Dor, 18(3), 217-24. http://dx.doi.org/10.5935/1806-0013.20170105.

Santos, M. D. L., \& Galdeano, L. E. (2009). Traço e Estado de ansiedade de estudantes de enfermagem na realização de uma prova prática. Rev. Min. Enferm,1(1), 76-83.

Sousa, E. F., Moreira, T. R., \& Santos, L. H. G. (2016). Correlação do nível de ansiedade e da qualidade de vida com os sinais e sintomas da disfunção temporomandibular em universitário. Clipe Odonto-UNITAU., 8(1), 16-21.

Spielberger, C. D., Gorsuch, R. L., \& Lushene, R. E. (1970). Manual for the statetrait anxiety inventory ("self-evaluatin questionaire"). California: ConsultingPsychologists.

Torres, F., Campos, L. G., Fillipini, H. F., Weigert, K. L., \& Vecchia, G. F. D. (2012). Efeitos dos tratamentos fisioterapêutico e odontológico em pacientes com disfunção temporomandibular. Fisioter. Mov. 25(1), 117-25. 